\title{
Smartphone-Based Meditation for Myeloproliferative Neoplasm Patients: Feasibility Study to Inform Future Trials
}

Jennifer Huberty ${ }^{1}$, PhD; Ryan Eckert ${ }^{2}$, MS; Linda Larkey ${ }^{3}$, PhD; Jonathan Kurka ${ }^{1}$, PhD; Sue A Rodríguez De Jesús ${ }^{1}$, $\mathrm{PhD}$; Wonsuk Yoo ${ }^{1}, \mathrm{PhD}$; Ruben Mesa ${ }^{2}, \mathrm{MD}$

\footnotetext{
${ }^{1}$ College of Health Solutions, Arizona State University, Phoenix, AZ, United States

${ }^{2}$ Mays Cancer Center, University of Texas Health MD Anderson, San Antonio, TX, United States

${ }^{3}$ College of Nursing and Health Innovation, Arizona State University, Phoenix, AZ, United States
}

Corresponding Author:

Jennifer Huberty, PhD

College of Health Solutions

Arizona State University

500 North 3rd St

Phoenix, AZ, 85004

United States

Phone: 14023011304

Email: jhuberty@asu.edu

\section{Abstract}

Background: Myeloproliferative neoplasm (MPN) patients often report high symptom burden that persists despite the best available pharmacologic therapy. Meditation has gained popularity in recent decades as a way to manage cancer patient symptoms.

Objective: The aim of this study was to examine the feasibility of 2 different consumer-based meditation smartphone apps in MPN patients and to examine the limited efficacy of smartphone-based meditation on symptoms compared with an educational control group.

Methods: Patients $(\mathrm{n}=128)$ were recruited nationally through organizational partners and social media. Eligible and consented patients were enrolled into 1 of 4 groups, 2 of which received varying orders of 2 consumer-based apps (10\% Happier and Calm) and 2 that received one of the apps alone for the second 4 weeks of the 8-week intervention after an educational control condition. Participants were asked to perform $10 \mathrm{~min}$ of meditation per day irrespective of the app and the order in which they received the apps. Feasibility outcomes were measured at weeks 5 and 9 with a Web-based survey. Feasibility outcomes were acceptability, demand, and limited efficacy for depression, anxiety, pain intensity, sleep disturbance, sexual function, quality of life, global health, and total symptom burden.

Results: A total of 128 patients were enrolled across all 4 groups, with 73.4\% (94/128) patients completing the intervention. Of the participants who completed the $10 \%$ Happier app, $61 \%(46 / 76)$ enjoyed it, $66 \%(50 / 76)$ were satisfied with the content, and $77 \%$ (59/76) would recommend to others. Of those who completed the Calm app, 83\% (56/68) enjoyed it, 84\% (57/68) were satisfied with the content, and 97\% (66/68) would recommend to others. Of those who completed the educational control, $91 \%$ (56/61) read it, 87\% (53/61) enjoyed it, and 71\% (43/61) learned something. Participants who completed the 10\% Happier app averaged 31 (SD 33) min/week; patients completing the Calm app averaged 71 (SD 74) min/week. 10\% Happier app participants saw small effects on anxiety $(P<.001 d=-0.43)$, depression $(P=.02 ; d=-0.38)$, sleep disturbance $(P=.01 ; d=-0.40)$, total symptom burden $(P=.13 ; d=-0.27)$, and fatigue $(P=.06 ; d=-0.30)$, and moderate effects on physical health $(P<.001 ; d=0.52) . C a l m$ app participants saw small effects on anxiety $(P=.29 ; d=-0.22)$, depression $(P=.09 ; d=-0.29)$, sleep disturbance $(P=.002 ; d=-0.47)$, physical health $(P=.005 ; d=0.44)$, total symptom burden $(P=.13 ; d=-0.27)$, and fatigue $(P=.13 ; d=-0.27)$. Educational control participants $(\mathrm{n}=61)$ did not have effects on any patient-reported outcome except for a moderate effect on physical health $(P<.001$; $d=0.77$ ).

Conclusions: Delivering meditation via the Calm app is feasible and scored higher in terms of feasibility when compared with the $10 \%$ Happier app. The Calm app will be used to implement a randomized controlled trial, testing the effects of meditation on symptom burden in MPNs.

Trial Registration: ClinicalTrials.gov NCT03726944; https://clinicaltrials.gov/ct2/show/NCT03726944 (Archived by WebCite at http://www.webcitation.org/77MVdFJwM) 
(JMIR Form Res 2019;3(2):e12662) doi: 10.2196/12662

\section{KEYWORDS}

mindfulness; meditation; smartphone; mHealth; cancer; quality of life

\section{Introduction}

Myeloproliferative neoplasms (MPNs) are a rare blood cancer with the most common subtypes including polycythemia vera, essential thrombocythemia, and myelofibrosis. Those afflicted with MPN report a high prevalence of fatigue, anxiety, depression, pain, and sleep disturbance [1,2]. The vast majority of MPN patients (81\% to 95\%) report fatigue as the most prevalent and severe symptom reducing physical, social, and cognitive functioning as well as quality of life [2-4]. MPN patients often have a favorable life expectancy, with as many as $60 \%$ of patients living up to 15 years after diagnosis. Many patients even have the same life expectancy as the general population $[5,6]$. This means that MPN patients live much of their lives with fatigue, other symptoms, and the associated detrimental effects on overall functioning and quality of life $[3,7]$.

There has been significant advancement in the pharmacological treatment of MPNs; however, for most patients, residual symptoms (ie, fatigue, anxiety, depression, pain, and sleep disturbance) persist, even with active pharmacologic therapy $[8,9]$. Additionally, standard-of-care treatments for MPN, including medication/drug therapy (ie, hydroxyurea), phlebotomy, and bone marrow transplant (the only curative therapy, reserved for those with severely reduced life expectancy), are associated with worsened symptoms and a reduced quality of life [10]. There is a need for research examining adjuvant, nonpharmacologic approaches (eg, mindfulness-based therapies) for MPN patients that may improve symptoms and that are not accompanied by negative side effects.

Extensive research in recent decades demonstrates the benefits of mindfulness-based therapies for alleviating fatigue, anxiety, depression, and sleep disturbance in both cancer and noncancer populations [11-16]. Mindfulness meditation has gained increasing attention as a complementary therapy for cancer patients, particularly for alleviating psychological comorbidities associated with cancer and its treatment [17]. Mindfulness meditation is the practice of moment-to-moment awareness in which the person purposefully focuses on the present moment without judgment [18]. Mindfulness meditation (hereinafter referred to as meditation) may be a potentially beneficial complementary therapy that improves MPN symptoms through increased nonjudgmental awareness of thoughts, feelings, and body sensations. These increases in awareness may help reduce feelings of anxiety and depression and may also help alleviate sleep disturbances resulting from sleep-interfering cognitive processes (ie, ruminating thoughts while trying to sleep) $[12,16]$. However, there has been minimal research investigating the effects of mindfulness meditation as a complementary therapy in hematological cancer patients and, specifically, no research conducted on MPN patients [19].
There has been a growing trend in recent decades toward digital (ie, Web-based and smartphone-based) interventions for health care access and to improve well-being. MPN patients may be a population that can greatly benefit from a digital intervention owing to (1) the rarity of MPNs, (2) the lack of specialized treatment centers in the United States, (3) the increasing prevalence of smartphone ownership in the United States, and (4) reported limitations to participation in in-person interventions among cancer patients. In a recent study, it was demonstrated that only 64\% (18/28) and 29\% (8/28) of MPN patients received their specialty care in the same state or city, respectively, in which they resided [20]. Additionally, the vast majority of Americans (more than $77 \%$ ) own a smartphone, with $62 \%$ using their smartphones to get health information and 30\% accessing a Web-based/mobile app to get educational content [21-23]. Finally, cancer patients report barriers to participating in in-person interventions, including fatigue, pain, and transportation/scheduling difficulties [24]. This asserts MPN patients as a population with the potential to benefit from digital meditation because they are more readily accessible and implemented with fewer resources than in-person meditation interventions.

As of January 2018, there were almost 6 million consumer-based smartphone apps available across both the Google Play Store and Apple's App Store, the 2 leading app stores in the world [25]. Of these, there are over 500 mindfulness/meditation-based smartphone apps available [26]. Despite the large number of consumer-based smartphone mindfulness/meditation apps available, very little research has investigated the feasibility and efficacy of these apps. Components and features across meditation apps may be more feasible (ie, acceptable, demanded, and practical) than others, potentially impacting user outcomes such as frequency of use and long-term compliance [27]. Although research is limited investigating the desired features of a consumer-based smartphone meditation app, there are reports about desired features in physical activity smartphone apps (eg, automatic tracking of activity, tracking of progress toward goals, and integrated features) [28] and across health behavior change apps more broadly (eg, accuracy of information, security of personal information, effort required to track progress, and immediate effects on mood) that help to inform interventions [29]. However, no research has specifically investigated the feasibility and desirability of one meditation app compared with another, especially to inform future interventions in specific populations.

Owing to the potential beneficial effects that meditation may have on residual MPN symptoms, the potential ease of delivery to a rare cancer such as MPNs, and the potential for dissemination of a consumer-based smartphone meditation app across other cancer populations, there is a need to examine meditation delivered via consumer-based smartphone apps in MPN patients. Therefore, the aim of this study was to examine the feasibility of 2 different consumer-based meditation 
smartphone apps in MPN patients and to examine the limited efficacy of smartphone-based meditation on symptoms compared with an educational control group. According to Bowen et al [30], limited efficacy refers to testing the intervention with limited statistical power and is appropriate for feasibility studies.

\section{Methods}

This study was approved by the Institutional Review Board at Arizona State University, and all participants signed an informed consent before participating in the study. All personal information was collected electronically and was stored in a password-protected computer at Arizona State University. The study was retrospectively registered with ClinicalTrials.gov.

\section{Study Design}

This was a 4-group randomized controlled trial (RCT) with a cross-over design. A convenience sample of eligible and consented MPN patients was recruited and enrolled into 1 of 4 groups with varying order of receiving 2 consumer-based meditation smartphone apps (ie, 10\% Happier app or Calm app) in 4-week increments over 8 weeks. Group 1 received the $10 \%$ Happier app followed by the Calm app; Group 2 received the Calm app followed by the $10 \%$ Happier app; Group 3 received educational control followed by the 10\% Happier app; and Group 4 received educational control followed by the Calm app. We chose the 4-group design to maximize the number of participants who use both apps. We did not have a washout period because the primary goal was to assess feasibility, not efficacy.

We used the Calm app for this study because Calm is one of the most popular consumer-based mobile apps (ie, Apple's app of the year in 2017), the lead author developed a relationship with Calm to conduct research using the app, and Calm agreed to provide the memberships to the app and share the tracking data with the research team without cost. The $10 \%$ Happier app was chosen because they were one of the competing meditation mobile apps of Calm and they agreed to provide the memberships to the app and share the tracking data with the research team without cost. Additionally, both smartphone apps are available across all major smartphone platforms (ie, Android and $\mathrm{iOS})$.

\section{Recruitment and Enrollment}

MPN patients $(n=128)$ were recruited online through MPN organizational partners with a flier outlining the study and its requirements. The study was advertised as a smartphone app meditation study. MPN patients interested in the study were asked to complete a Web-based eligibility questionnaire administered via Qualtrics. Textbox 1 describes the eligibility criteria. Researchers checked the eligibility questionnaires as they were completed and emailed patients their eligibility status. Eligible patients were invited to participate in a 20 -min phone appointment in which the study details and informed consent were described in detail. MPN patients who completed the intake appointment were then sent an informed consent electronically via Qualtrics that included a place for their electronic signature. Ineligible patients received an email stating their ineligibility status as well as links to both consumer-based apps used in the study, in case the ineligible participant was interested in trying meditation.

Upon receipt of the completed informed consent, participants were randomly assigned to one of 4 groups. A group assignment list was computationally generated through randomizer.org by a research assistant to randomly allocate participants to one of 4 equally sized groups before study commencement This pre-generated list was then used by the same research assistant to place eligible, consented MPN patients into their group assignment in the order in which they consented to participate. Randomized participants were provided with a welcome email that contained (1) a welcome letter introducing them to the study, (2) a calendar detailing important study dates, and (3) instructions specific to the first assigned condition (ie, $C B$ app, Calm app, or educational control) to be introduced for the first 4 weeks. After participants completed the first of their 24 -week conditions (ie, $10 \%$ Happier app, Calm app, or control), they were provided with another email that included instructions specific to their final condition (ie, 10\% Happier app, Calm app, or educational control).

Textbox 1. Eligibility criteria.

Inclusion criteria:

- Had a diagnosis of essential thrombocythemia, polycythemia vera, or myelofibrosis identified by the treating physician

- $\quad$ Owned a mobile smartphone and were willing to download and use a meditation app (ie, 10\% Happier or Calm)

- Could read and understand English

- Were aged 18 years or older

- Were willing to be randomized to one of 4 groups: (1) 10\% Happier 4 weeks/Calm 4 weeks, (2) Calm 4 weeks/10\% Happier app 4 weeks, (3) educational material 4 weeks/10\% Happier app 4 weeks, and (4) educational material 4 weeks/Calm 4 weeks

Exclusion criteria:

- $\quad$ Engaged in $\geq 10 \mathrm{~min} /$ day of meditation on $\geq 5$ days/week for the past 6 months

- $\quad$ Engaged in $\geq 60 \mathrm{~min} /$ week of tai chi, qigong, or yoga each week

- $\quad$ Utilized either the $10 \%$ Happier app or the Calm app

- $\quad$ Resided outside of the United States 


\section{Procedures}

All participants were instructed to listen to the app's introduction to meditation and, following this, were instructed to participate in a 10-min meditation each day, which they selected from the app's library of meditations. The dose for the intervention was chosen because (1) the Calm and 10\% Happier app currently offer 10-min meditations (daily and series meditations), thus representing the length of time users are most likely to meditate using the app; and (2) to date, the ideal dose for mindfulness meditation interventions has not been established and effective mindfulness meditation interventions have ranged from 10-min sessions to 2-hour sessions and from one day per week to a daily practice [31-33]. Practical guidelines recommend that beginners start with short meditations lasting between $10 \mathrm{~min}$ and $30 \mathrm{~min}$ per session [34].

All study participants were asked to complete Web-based surveys at baseline, Week 5, and Week 9 via Qualtrics to assess feasibility outcomes (described in detail below), including study satisfaction and patient-reported outcomes. The survey consisted of 40 to 50 questions (depending on the condition the participant just completed and whether it was at baseline or post-condition) and was developed and tested internally among the research team for usability and functionality before use in the study. At the end of each 4-week condition, participants were emailed a unique survey link specific to their study identification number and were asked to complete the survey within 24 to 48 hours, if possible. Responses were collected electronically in Qualtrics and were then entered manually into a de-identified Excel spreadsheet for later data analysis. All participants received the same surveys in the same question order each time. The only difference between surveys was the wording of the satisfaction questions based on which condition they completed. Participants were able to scroll back through their survey responses by navigating back and forth through pages with a back button, giving them the flexibility to revise questions if they needed. Participants were only allowed to complete the survey once, and after it was submitted, they were not allowed access back into the survey.

Additionally, all study participants were instructed to wear a Fitbit (Fitbit Inc) device on their nondominant wrist throughout the 8-week intervention to measure physical activity and sleep; however, these data are not reported here. The intervention was 8 weeks because this is an appropriate length of time for a feasibility study in which the primary purpose was not to determine efficacy. Similar lengths have been used in other feasibility studies [30,35].

\section{Description of the Apps and the Control}

\section{0\% Happier App}

The 10\% Happier app's introduction to meditation incorporated basic information for those new to meditation. Daily meditations were selected from a library of meditations included within the app. Each of the meditations had a different focus (eg, grief, gratitude, choice, and letting go) and were approximately 10 to 12 min in length.

\section{Calm App}

The Calm app's introduction to meditation incorporated basic educational information for those new to meditation while introducing brief experiential practices. Daily meditations were called the Daily Calm and were new and unique, provided by the app each day. The daily meditations had a different focus (eg, practicing patience, loving kindness, and gratitude) and were approximately 10 to $12 \mathrm{~min}$ in length. Meditations were also selected from a library of meditations with the app.

\section{Educational Control}

The control condition was provided with a 7-page educational handout that was developed by the research team before the study. The handout addressed MPN patient fatigue (eg, what causes fatigue?) as well as examples of and information related to evidence-based fatigue management strategies.

\section{Feasibility Outcomes}

Feasibility (ie, acceptability, demand, and limited-efficacy testing) was defined according to Bowen et al [30]. Acceptability was measured with an investigator-developed set of satisfaction survey questions at Week 5 and Week 9 after participants completed each of their 4-week group assignments. Satisfaction questions were the same for all 3 conditions, with the exception of the wording that was altered slightly so that the question fit each condition. For example, a question asked in the satisfaction survey included: "How likely are you to continue using the (insert condition here) on a 1 to 5 scale ( 1 being very poor; 5 being excellent)". Benchmarks for acceptability included $\geq 70 \%$ satisfaction with the apps' content, $\geq 70 \%$ intending to continue using the app, $\geq 70 \%$ enjoying using the app, and $\geq 70 \%$ recommending it for other MPN patients. Demand was measured using adherence to the meditation intervention. Meditation participation was tracked by the smartphone app developers and reported weekly to the research team. Reports included (1) the date and time of each meditation participated in, (2) the title of the meditation, and (3) the duration of participation (ie, the time spent viewing the meditation) for each participant. Adherence benchmarks were defined as an average of $\geq 49$ $\mathrm{min} /$ week of meditation across all participants (ie, $\geq 70 \%$ of prescribed meditation). There is little research suggesting appropriate benchmarks for acceptability and demand [30]. We based our benchmarks on a recently published methods paper for a National Institute of Health-funded study for the feasibility of a Web-based yoga intervention [36]. Patient-reported outcomes were assessed in all study participants via a Qualtrics questionnaire at baseline, Week 5, and Week 9. Demographics and MPN-related health history were included within the baseline questionnaire only. The National Institutes of Health Patient Reported Outcomes Measurement Information System (NIH PROMIS) and the MPN Symptom Assessment Form (MPN-SAF) were used to measure patient-reported outcomes. MPN-SAF measures included total symptom score and fatigue (Question 1 on MPN-SAF). NIH PROMIS measures included anxiety, depression, pain intensity, sleep disturbance, sexual function, global health, and quality of life (Question 2 on the global health scale). Table 1 describes each of these outcome measures. 
Table 1. Summary of self-report outcome measures.

\begin{tabular}{ll}
\hline Measure, outcome & Scoring \\
\hline MPN SAF & \\
Total symptom score & 10 items (0-10 scale); total score range of 0-100 with higher score indicating worse symptom burden \\
Fatigue & Item \#1 from MPN SAF; 0-10. Scale with higher score indicating more fatigue \\
NIH PROMIS & \\
Anxiety & $\begin{array}{l}\text { 8-item measure with each question asked on a 1-5 scale; total cumulative raw score converted to standardized t-score; } \\
\text { higher t-score represents more of the construct being measured } \\
\text { 8-item measure with each question asked on a 1-5 scale; total cumulative raw score converted to standardized t-score; } \\
\text { higher t-score represents more of the construct being measured }\end{array}$ \\
Pain intensity & $\begin{array}{l}\text { 3-item measure with each question asked on a 1-5 scale; total cumulative raw score converted to standardized t-score; } \\
\text { higher t-score represents more of the construct being measured }\end{array}$ \\
Sleep disturbance & $\begin{array}{l}\text { 8-item measure with each question asked on a 1-5 scale; total cumulative raw score converted to standardized t-score; } \\
\text { higher t-score represents more of the construct being measured }\end{array}$ \\
Sexual function & $\begin{array}{l}\text { 11-item measure with each question asked on a 1-5 scale; total cumulative raw score converted to standardized t-score; } \\
\text { higher t-score represents more of the construct being measured }\end{array}$ \\
Global health & $\begin{array}{l}\text { 10-item measure with each question asked on a 1-5 scale; total cumulative raw score converted to standardized t-score; } \\
\text { higher t-score represents more of the construct being measured }\end{array}$ \\
Quality of life & Item \#2 on the Global Health scale; scored on a 1-5 scale with a higher raw score indicating a lower quality of life
\end{tabular}

${ }^{\mathrm{a}}$ MPN-SAF: MPN Symptom Assessment Form.

${ }^{b}$ NIH PROMIS: National Institutes of Health Patient Reported Outcomes Measurement Information System.

\section{Data Analysis}

Descriptive analyses were performed for baseline demographic characteristics using means and SDs of continuous data and using frequencies and proportions of discrete data for the 2 apps and 2 control groups, and dropouts. To test the limited efficacy of the intervention, a series of analysis of covariance analyses were performed for each of the NIH PROMIS outcomes using raw scores on measures of pain intensity, anxiety, depression, sleep disturbance, sexual function and discomfort indicators, global health, and quality of life. The analysis was adjusted for baseline PROMIS levels and covariates of the group membership (sequence effects), gender, education, and marital status. Race was not considered as a covariate as less than $5 \%$ of the sample was non-white Dunnett's post hoc tested differences between treatment outcomes compared with baseline. A 2-tailed alpha error of .05 was the threshold for statistical significance. In addition, effect sizes (Cohen $d$ ) were calculated and classified as small $(d=0.2)$, medium $(d=0.5)$, and large $(d=0.8)$ to examine differences at postcondition time. A negative effect size indicates the outcome measure decreased between the baseline and postcondition measure [37]. All analytical and visual evidence including appropriate statistics, $P$ values, and graphs was reported using Statistical Analysis Software (SAS Institute, version 9.4) and Microsoft Excel (2016). A $P$ value of $<.05$ was considered statistically significant.

\section{Results}

\section{Recruitment and Enrollment}

A total of $289 \mathrm{MPN}$ patients were recruited and completed the eligibility survey between July 31 and October 18, 2017 (ie, two-and-a-half months). Of the $289 \mathrm{MPN}$ patients who completed the eligibility survey, $33.2 \%(96 / 289)$ were ineligible and $44.2 \%$ (128/289) signed the informed consent and were enrolled into the study (see Multimedia Appendix 1). Eligible participants were enrolled into the study in the order they completed the eligibility questionnaire. A total of $73.4 \%$ (94/128) patients across all 4 groups completed the intervention. See Table 2 for protocol adherence and completion rates across groups. The results below are reported for participants who completed the intervention (ie, completed both postcondition surveys at Week 4 and Week 8). 
Table 2. Protocol adherence.

\begin{tabular}{llll}
\hline Group & $\mathrm{N}$ (signed informed consent) & Completed post-Week 4 survey ${ }^{\mathrm{a}}, \mathrm{n}(\%)$ & ${\text { Completed post-Week } 8 \text { survey }{ }^{\mathrm{a}}, \mathrm{n}(\%)}_{26(79)}$ \\
\hline 10\% Happier followed by Calm & 33 & $28(85)$ & $26(81)$ \\
Calm followed by $10 \%$ Happier & 32 & $24(69)$ & $24(69)$ \\
Control followed by $10 \%$ Happier & 35 & $18(64)$ & $18(64)$ \\
Control followed by Calm & 28 & $96(75)$ & $94(73)$ \\
Total & 128 & & 26 \\
\hline
\end{tabular}

${ }^{\text {a }}$ These surveys include satisfaction questions related to the completed 4-week group assignment.

There were no significant differences between groups on any demographic variable. Across all participants $(\mathrm{n}=128)$, average age was 58 (SD 12) years $\left(F_{1,126}=2.45 ; P=.12\right)$ and average body mass index was $27(\operatorname{SD} 6) \mathrm{kg} / \mathrm{m}^{2}\left(F_{1,123}=.28 ; P=.60\right)$. The majority were female $\left(104 / 128 ; \chi_{3}^{2}=4.0 ; P=.26\right)$, white $(123 / 128$; $\left.\chi_{9}^{2}=9.8 ; P=.36\right)$, well-educated with a bachelor's education or higher (79/128; $\left.\chi_{15}^{2}=15.7 ; P=.40\right)$, and married (95/128; $\left.\chi_{15}^{2}=14.4 ; P=.49\right)$. In addition, the most common diagnosis among all participants was Essential Thrombocythemia (54/128) followed by Polycythemia Vera (48/128) and Myelofibrosis (26/128). Most participants had been diagnosed with their MPN for more than 3 years $(81 / 128)$.

\section{Feasibility Outcomes}

\section{Acceptability}

See Multimedia Appendix 2 for satisfaction survey responses. Of the participants who used the 10\% Happier app, 61\% (46/76)

Figure 1. Weekly meditation participation. enjoyed it, 66\% (50/76) were satisfied with the content, and $77 \%$ (59/76) would recommend it to others. Of those who completed the Calm app, 83\% (56/68) enjoyed it, 84\% (57/68) were satisfied with the content, and 97\% (66/68) would recommend it to others. Of those who completed the educational control, 91\% (56/61) read it, 87\% (53/61) enjoyed it, and 71\% (43/61) learned something.

\section{Demand}

Participants who completed the 10\% Happier app averaged 31 (SD 33) $\mathrm{min} /$ week and 30\% (23/76) averaged $\geq 49 \mathrm{~min} /$ week of meditation, whereas participants who completed the Calm app averaged 71 (SD 74) min/week and 56\% (38/68) averaged $\geq 49$ $\mathrm{min} /$ week of meditation. See Figure 1 depicting average weekly meditation minutes completed by participants.
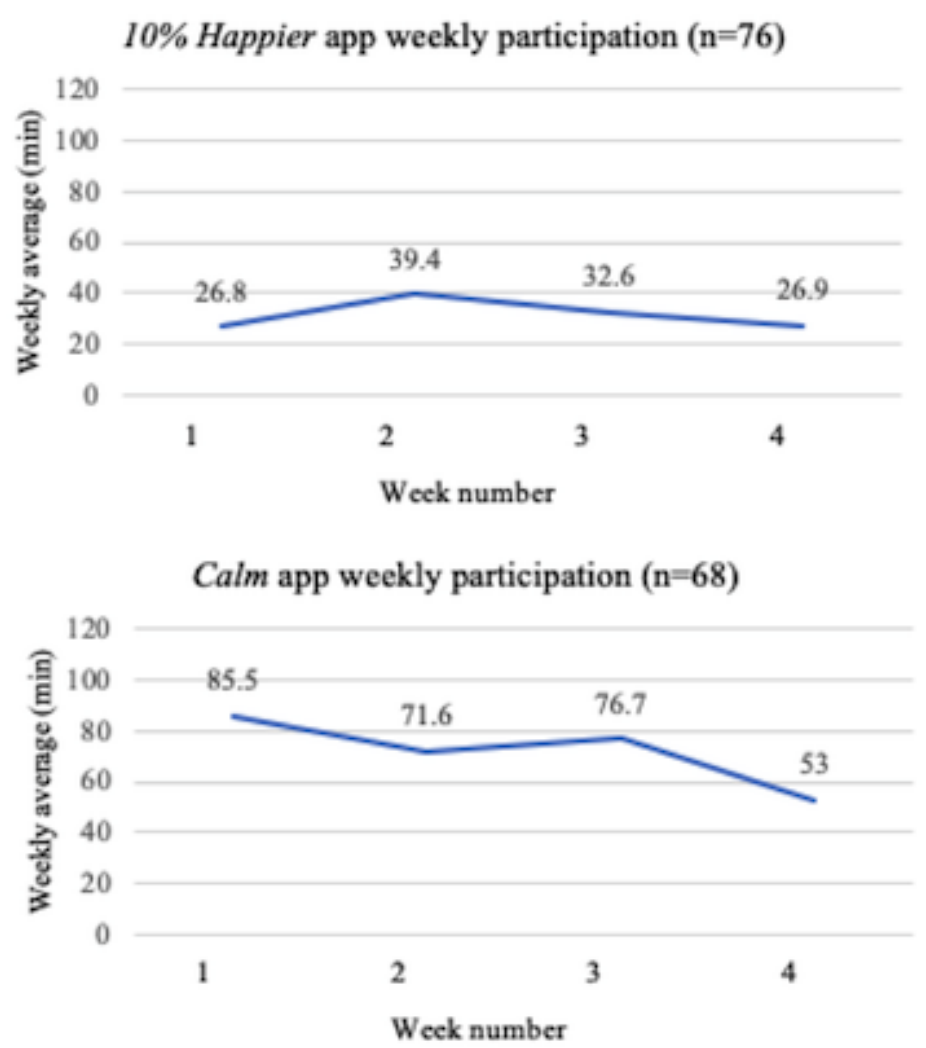


\section{Limited Efficacy Testing}

Mean differences in patient-reported outcomes from baseline to postcondition are reported in Multimedia Appendix 3. After completing 4 weeks of meditation using the 10\% Happier app $(\mathrm{n}=76)$, significant differences were found between baseline and postcondition for anxiety $(P<.001 ; d=-0.43)$, depression $(P=.02 ; d=-0.38)$, sleep disturbance $(P=.01 ; d=-0.40)$, and physical health $(P<.001 ; d=0.52)$. Mental health $(P=.07$; $d=-0.30)$ and fatigue $(P=.06 ; d=-0.30)$ were approaching significance. Although not statistically significant, a small-to-medium effect size and the $95 \% \mathrm{CI}$ indicate differences in total symptom burden ( $d=-0.27$; 95\% CI -11.75 to -0.09$)$.

After completing 4 weeks of meditation using the Calm app $(n=68)$, significant differences were found between baseline and postcondition for sleep disturbance $(P=.002 ; d=-0.47)$, vaginal discomfort $(P=.03 ; d=-0.36)$, and physical health $(P=.005 ; d=0.44)$. Small-to-medium effects and $95 \% \mathrm{CI}$ indicated differences for measures of depression $(P=.09$; $d=-0.29 ; 95 \% \mathrm{CI}-3.49$ to -0.14$)$, total symptom burden $(P=.13$; $d=-0.27 ; 95 \% \mathrm{CI}-11.81$ to -0.11$)$, and fatigue $(P=.13 ; d=-0.27$; $95 \% \mathrm{CI}-1.68$ to -0.02$)$, but were not significant.

After completing 4 weeks of the educational control $(n=61)$, the only effect seen between baseline and postcondition was for physical health $(P<.001 ; d=0.77)$.

\section{Discussion}

\section{Principal Findings}

The purpose of this study was to examine the feasibility of 2 different smartphone-based meditation apps in MPN patients and to examine the limited efficacy of smartphone-based meditation on symptoms compared with an educational control group. The findings of this study will inform the app to be used for a larger RCT to test for efficacy. We have identified the Calm app as being feasible for our future RCT as it met nearly all of our a priori feasibility criteria (ie, demand, acceptability, and limited-efficacy testing).

Our findings suggest that the Calm app had higher demand than the $10 \%$ Happier app. Overall, the average weekly meditation across participants that completed Calm was greater than the prescribed $70 \mathrm{~min} /$ week and over half averaged $\geq 49 \mathrm{~min} /$ week of meditation (ie, demand cutoff criterion). Comparatively, those who participated in the $10 \%$ Happier app only averaged $31 \mathrm{~min} /$ week of meditation and less than a third averaged $\geq 49$ $\mathrm{min} /$ week of meditation. The adherence rates of the Calm app group are promising findings related to the potential for delivering meditation using a consumer-based smartphone app in MPN patients.

The adherence rates demonstrated by those completing Calm in this study are better than adherence rates of other smartphone-based meditation app studies [38,39]. A recent study conducted by Economides et al [38] investigated the effects of meditation delivered using the Headspace app on stress, affect, and irritability in novice meditators and found that participants $(n=41)$ averaged approximately $44 \mathrm{~min} /$ week of meditation when asked to complete a total of 10 introductory 10-min meditations as they desired. Participants used the app for a short duration (approximately 16 days). Another recent study conducted by Bostock et al [39] that examined the effects of a 45-day Headspace meditation app intervention on work stress and well-being in healthy workers found that participants averaged approximately $42 \mathrm{~min} /$ week of meditation. Our study was 28 days in length (ie, the length participants were asked to use each app) and the Calm app group averaged approximately $71 \mathrm{~min} /$ week. It is not clear why our study had better participation in meditation as compared with the aforementioned studies. However, Calm may have characteristics (eg, app layout, presentation of content, and variety of content) within the app that make it more likely to be accepted and used by participants [28,29] when compared with Headspace or other meditation apps (ie, 10\% Happier app).

Those who completed Calm indicated that they enjoyed it (83\%), were satisfied with the content $(84 \%)$, and would recommend it to others $(97 \%)$, each of which meets our benchmarks for acceptability. Of those that used the 10\% Happier app, $61 \%$ enjoyed it, $66 \%$ were satisfied with the content, and $77 \%$ would recommend to others. With the exception of those that would recommend it to others, the 10\% Happier app fell short of meeting benchmarks for acceptability. Self-report responses from those in Calm indicate the acceptability of the app compared with the $10 \%$ Happier app. We conducted qualitative interviews related to the content and preferences for each of the apps; these data are being reported elsewhere [40]. However, briefly, the reasons Calm may have been more accepted than the 10\% Happier app were related to audio features (ie, the narrator voice was soothing and calming background sounds available) and other features such as pictures, stories, and a wide range of meditation topics.

Research with physical activity and other health behavior apps suggests automatic tracking of the specific activity, tracking of progress toward goals, and integrated features (eg, syncing with social media and connecting with music apps) are features most liked by users [28]. Both the Calm app and the $10 \%$ Happier app share similarities in terms of their tracking features consistent with those reported in the literature as desired features of smartphone apps [28,29,41]. However, the Calm app automatically displays the tracking information following participation in meditation (pops up onto the screen) and includes minutes meditated, days in a row of mediating (ie, streak), and a calendar that highlights the days in which the app was used. To see the tracking of meditation in the $10 \%$ Happier app, the user must navigate to their profile to look up the statistics that are displayed as days and minutes meditated. The differences in the way that the tracking is offered to the user may contribute to the differences in the acceptability of the apps. More exploration about the type of tracking and how participants prefer to interact with a tracking mechanism within a mobile app, specifically, a mediation app, is warranted.

Unlike the $10 \%$ Happier app, Calm allows users to immediately share their meditation statistics (eg, number of meditations and time spent in meditation) through social media platforms (eg, Facebook) or text messages and emails (called share status). Even though we did not measure the use of the share status, the offering of this on the app may also contribute to its enhanced acceptability. Recent research has shown that 
participants like to share progress and that sharing progress updates and interacting within an online social platform may help increase feelings of accountability and may improve adherence rates in interventions $[42,43]$. In a recent qualitative study, participants with diverse health-related goals that shared updates on their progress via social media reported sharing their updates helped contribute to their accountability [42].

Both the Calm and 10\% Happier apps include a variety of content for users outside of just listening to meditation tracks. Calm includes sleep stories or sleep meditations to help users fall asleep. They also offer Calm Breathe, Calm Music, Calm Body, and relaxing scenes and nature sounds. Calm has masterclasses to educate participants about topics such as mindful eating, gratitude, and the importance of rest. The $10 \%$ Happier app offers some similar content but is organized and delivered differently. For example, the content is organized on the bottom tool bar and users scroll to find the content they want to use (Calm organizes with a toolbar but also includes other screens for organization of content). The sleep content is only in the form of meditations (not sleep stories). Education for participants is in the form of courses with a meditation to follow-up the content (eg, a short 3 to 5 min lecture-based video followed by a meditation). On the basis of our findings, the way in which the Calm app organizes and delivers their content may be more appealing as compared with the 10\% Happier app. Future studies are warranted to determine what users specifically find most acceptable related to how content is organized and offered.

We observed limited efficacy on symptoms across both apps. Of note are the small effects observed in both apps on anxiety, depression, sleep disturbance, and total symptom burden. Although this is the first meditation study to be conducted in MPN patients, Huberty et al [20] identified that 12 weeks of Web-based yoga (60 min/week) had limited efficacy on anxiety, depression, sleep disturbance, and total symptom burden in MPN patients. Meditation and yoga differ in the sense that yoga includes a component of physical postures (asanas), but yoga does contain a meditation component and it is known that some of yoga's benefits come from the meditation [44-48]. Meditation has been shown to improve anxiety, depression, and sleep disturbance symptoms in both cancer and noncancer populations through increased nonjudgmental awareness of thoughts, feelings, and body sensations [12,16]. Therefore, it is not surprising that this study showed limited efficacy in only 4 weeks on anxiety, depression, and sleep disturbances across both meditation apps. Studies of a longer duration may show larger effects. Our findings are preliminary in nature and RCTs powered for effectiveness are needed to determine the effects of consumer-based smartphone meditation on MPN patients.

\section{Limitations}

There are limitations to this study that should be noted. First, our sample was disproportionately female (ie, $81 \%$ in this study vs approximately $53 \%$ female being typical of the MPN population [49]) and white (ie, 123/128, 96\%). Second, we did not have a washout period in between each condition within the group assignments (ie, lack of washout time period between apps or between control and app conditions). As this was a feasibility study and we were not determining efficacy, a washout period was not necessary [50]. Finally, it is likely that the study findings represent those already motivated to use smartphones for health-related purposes. However, the literature suggests smartphone use is popular among cancer patients overall and that there is a large interest in accessing supportive care via smartphones in cancer patients [51,52]. Importantly, future studies could determine the effects of a mobile app to improve health-related outcomes in cancer patients, especially when cancer patients are already using mobile apps.

\section{Conclusions}

Delivering smartphone meditation via the Calm app is feasible as it met most of our feasibility criteria (ie, demand, acceptability, and limited-efficacy testing) and scored higher in terms of feasibility when compared with another consumer-based app (ie, 10\% Happier app). Future RCTs are needed to examine meditation with the Calm app and its effects on MPN patient symptoms. The findings of this study will be used to inform the development of a National Institutes of Health R01 grant app for an RCT examining the efficacy of Calm on MPN patient symptoms.

\section{Acknowledgments}

This study was funded with philanthropic funds. The authors would like to acknowledge both $10 \%$ Happier and Calm for donating memberships for participants in this study.

\section{Conflicts of Interest}

RM reports research support by Incyte, Celgene, CTI BioPharma, Abbvie, and Genetech. In addition, RM acts as a consultant for Novartis, La Jolla, and Sierra Oncology. The authors do not report any additional conflicts of interest.

Editorial note: This randomized study was only retrospectively registered. The editor granted an exception from ICMJE rules mandating prospective registration of randomized trials because the risk of bias appears low and the study was considered formative. However, readers are advised to carefully assess the validity of any potential explicit or implicit claims related to primary outcomes or effectiveness, as retrospective registration does not prevent authors from changing their outcome measures retrospectively. 


\section{Multimedia Appendix 1}

Enrollment.

[PDF File (Adobe PDF File), 140KB-Multimedia Appendix 1]

\section{Multimedia Appendix 2}

Satisfaction Survey Responses.

[PDF File (Adobe PDF File), 70KB-Multimedia Appendix 2]

\section{Multimedia Appendix 3}

Patient-Reported Outcomes.

[PDF File (Adobe PDF File), 76KB-Multimedia Appendix 3]

\section{References}

1. Emanuel RM, Dueck AC, Geyer HL, Kiladjian J, Slot S, Zweegman S, et al. Myeloproliferative neoplasm (MPN) symptom assessment form total symptom score: prospective international assessment of an abbreviated symptom burden scoring system among patients with MPNs. J Clin Oncol 2012 Nov 20;30(33):4098-4103 [FREE Full text] [doi: 10.1200/JCO.2012.42.3863] [Medline: 23071245]

2. Mesa RA, Niblack J, Wadleigh M, Verstovsek S, Camoriano J, Barnes S, et al. The burden of fatigue and quality of life in myeloproliferative disorders (MPDs): an international internet-based survey of 1179 MPD patients. Cancer 2007 Jan 01;109(1):68-76 [FREE Full text] [doi: 10.1002/cncr.22365] [Medline: 17123268]

3. Mesa R, Miller CB, Thyne M, Mangan J, Goldberger S, Fazal S, et al. Myeloproliferative neoplasms (MPNs) have a significant impact on patients' overall health and productivity: the MPN Landmark survey. BMC Cancer 2016 Feb 27;16:167 [FREE Full text] [doi: 10.1186/s12885-016-2208-2] [Medline: 26922064]

4. Scherber R, Dueck AC, Johansson P, Barbui T, Barosi G, Vannucchi AM, et al. The Myeloproliferative Neoplasm Symptom Assessment Form (MPN-SAF): international prospective validation and reliability trial in 402 patients. Blood 2011 Jul 14;118(2):401-408 [FREE Full text] [doi: 10.1182/blood-2011-01-328955] [Medline: 21536863]

5. Hultcrantz M, Kristinsson SY, Andersson TM, Landgren O, Eloranta S, Derolf AR, et al. Patterns of survival among patients with myeloproliferative neoplasms diagnosed in Sweden from 1973 to 2008: a population-based study. J Clin Oncol 2012 Aug 20;30(24):2995-3001 [FREE Full text] [doi: 10.1200/JCO.2012.42.1925] [Medline: 22802311]

6. Tefferi A, Guglielmelli P, Larson DR, Finke C, Wassie EA, Pieri L, et al. Long-term survival and blast transformation in molecularly annotated essential thrombocythemia, polycythemia vera, and myelofibrosis. Blood 2014 Oct 16;124(16):2507-2513 [FREE Full text] [doi: 10.1182/blood-2014-05-579136] [Medline: 25037629]

7. Mehta J, Wang H, Fryzek JP, Iqbal SU, Mesa R. Health resource utilization and cost associated with myeloproliferative neoplasms in a large United States health plan. Leuk Lymphoma 2014 Oct;55(10):2368-2374. [doi: 10.3109/10428194.2013.879127] [Medline: 24450579]

8. Geyer H, Scherber R, Kosiorek H, Dueck AC, Kiladjian J, Xiao Z, et al. Symptomatic profiles of patients with polycythemia vera: implications of inadequately controlled disease. J Clin Oncol 2016 Jan 10;34(2):151-159. [doi: 10.1200/JCO.2015.62.9337] [Medline: 26598745]

9. Mesa R, Verstovsek S, Kiladjian J, Griesshammer M, Masszi T, Durrant S, et al. Changes in quality of life and disease-related symptoms in patients with polycythemia vera receiving ruxolitinib or standard therapy. Eur J Haematol 2016 Aug;97(2):192-200. [doi: 10.1111/ejh.12707] [Medline: 26608702]

10. Scotch A, Scherber R, Bruso M, Kosiorek H, Dueck A, Geyer H, et al. Myeloproliferative Neoplasm Quality of Life (MPN-QOL) study group: results from the MPN Experimental Assessment of Symptoms By Utilizing Repetitive Evaluation (MEASURE) trial. Blood 2017;128(22):1641 [FREE Full text]

11. Christodoulou G, Black DS. Mindfulness-based interventions and sleep among cancer survivors: a critical analysis of randomized controlled trials. Curr Oncol Rep 2017 Sep;19(9):60. [doi: 10.1007/s11912-017-0621-6] [Medline: 28748522]

12. Gong H, Ni CX, Liu YZ, Zhang Y, Su WJ, Lian YJ, et al. Mindfulness meditation for insomnia: a meta-analysis of randomized controlled trials. J Psychosom Res 2016 Dec;89:1-6. [doi: 10.1016/j.jpsychores.2016.07.016] [Medline: 27663102]

13. Hofmann SG, Sawyer AT, Witt AA, Oh D. The effect of mindfulness-based therapy on anxiety and depression: a meta-analytic review. J Consult Clin Psychol 2010 Apr;78(2):169-183 [FREE Full text] [doi: 10.1037/a0018555] [Medline: 20350028]

14. Johns SA, Brown LF, Beck-Coon K, Monahan PO, Tong Y, Kroenke K. Randomized controlled pilot study of mindfulness-based stress reduction for persistently fatigued cancer survivors. Psychooncology 2015 Aug;24(8):885-893 [FREE Full text] [doi: 10.1002/pon.3648] [Medline: 25132206]

15. Ong JC, Manber R, Segal Z, Xia Y, Shapiro S, Wyatt JK. A randomized controlled trial of mindfulness meditation for chronic insomnia. Sleep 2014 Sep 01;37(9):1553-1563 [FREE Full text] [doi: 10.5665/sleep.4010] [Medline: 25142566] 
16. Piet J, Würtzen H, Zachariae R. The effect of mindfulness-based therapy on symptoms of anxiety and depression in adult cancer patients and survivors: a systematic review and meta-analysis. J Consult Clin Psychol 2012 Dec;80(6):1007-1020. [doi: 10.1037/a0028329] [Medline: 22563637]

17. Musial F, Büssing A, Heusser P, Choi K, Ostermann T. Mindfulness-based stress reduction for integrative cancer care: a summary of evidence. Forsch Komplementmed 2011;18(4):192-202 [FREE Full text] [doi: 10.1159/000330714] [Medline: 21934319]

18. Kabat-Zinn J, Hanh T. Full Catastrophe Living: Using the Wisdom of Your Body and Mind to Face Stress, Pain, and Illness. New York, NY: Delta; 2009.

19. Salhofer I, Will A, Monsef I, Skoetz N. Meditation for adults with haematological malignancies. Cochrane Database Syst Rev 2016 Feb 03;2:CD011157. [doi: 10.1002/14651858.CD011157.pub2] [Medline: 26840029]

20. Huberty J, Eckert R, Gowin K, Mitchell J, Dueck AC, Ginos BF, et al. Feasibility study of online yoga for symptom management in patients with myeloproliferative neoplasms. Haematologica 2017 Dec;102(10):e384-e388. [doi: 10.3324/haematol.2017.168583] [Medline: 28596279]

21. Pew Research Center. 2018. Mobile Fact Sheet URL: http://www.pewinternet.org/fact-sheet/mobile/[WebCite Cache ID 730Ofjwns]

22. Pew Research Center. 2017. Key Trends in Social and Digital News Media URL: http://www.pewresearch.org/fact-tank/ 2017/10/04/key-trends-in-social-and-digital-news-media/ [accessed 2018-10-23] [WebCite Cache ID 73OOwkOHa]

23. Pew Research Center. 2015. US Smartphone Use in 2015 URL: http://www.pewinternet.org/2015/04/01/ us-smartphone-use-in-2015/[WebCite Cache ID 730OpJT1B]

24. van Waart H, van Harten WH, Buffart LM, Sonke GS, Stuiver MM, Aaronson NK. Why do patients choose (not) to participate in an exercise trial during adjuvant chemotherapy for breast cancer? Psychooncology 2016 Dec;25(8):964-970. [doi: 10.1002/pon.3936] [Medline: 26282696]

25. Statista. 2018. Number of apps available in leading app stores as of 3rd quarter URL: https://www.statista.com/statistics/ 276623/number-of-apps-available-in-leading-app-stores/[WebCite Cache ID 73OPIihnk]

26. Marlynn W. Psychology Today. 2017. What mindfulness app is right for you? URL: https://www.psychologytoday.com/ us/blog/urban-survival/201508/what-mindfulness-app-is-right-you [accessed 2018-10-23] [WebCite Cache ID 73OPbPIwJ]

27. Fischer K. Healthline. 2018. Best Meditation Apps of 2018 URL: https://www.healthline.com/health/mental-health/ top-meditation-iphone-android-apps [accessed 2018-10-23] [WebCite Cache ID 73OQpuk1M]

28. Rabin C, Bock B. Desired features of smartphone applications promoting physical activity. Telemed J E Health 2011 Dec;17(10):801-803. [doi: 10.1089/tmj.2011.0055] [Medline: 22010977]

29. Dennison L, Morrison L, Conway G, Yardley L. Opportunities and challenges for smartphone applications in supporting health behavior change: qualitative study. J Med Internet Res 2013;15(4):e86 [FREE Full text] [doi: 10.2196/jmir.2583] [Medline: 23598614]

30. Bowen DJ, Kreuter M, Spring B, Cofta-Woerpel L, Linnan L, Weiner D, et al. How we design feasibility studies. Am J Prev Med 2009 May;36(5):452-457 [FREE Full text] [doi: 10.1016/j.amepre.2009.02.002] [Medline: 19362699]

31. de Bruin EI, van der Zwan JE, Bögels SM. A RCT comparing daily mindfulness meditations, biofeedback exercises, and daily physical exercise on attention control, executive functioning, mindful awareness, self-compassion, and worrying in stressed young adults. Mindfulness (N Y) 2016;7(5):1182-1192 [FREE Full text] [doi: 10.1007/s12671-016-0561-5] [Medline: 27642375]

32. Grossman P, Niemann L, Schmidt S, Walach H. Mindfulness-based stress reduction and health benefits. A meta-analysis. J Psychosom Res 2004 Jul;57(1):35-43. [doi: 10.1016/S0022-3999(03)00573-7] [Medline: 15256293]

33. Moore AW, Gruber T, Derose J, Malinowski P. Regular, brief mindfulness meditation practice improves electrophysiological markers of attentional control. Front Hum Neurosci 2012;6:18 [FREE Full text] [doi: 10.3389/fnhum.2012.00018] [Medline: 22363278]

34. McDonald K. How to Meditate: A Practical Guide. Somerville, MA: Wisdom Publications; 2005.

35. Chittaro L, Vianello A. Evaluation of a mobile mindfulness app distributed through on-line stores: a 4-week study. Int J Hum Comput Stud 2016 Feb;86:63-80. [doi: 10.1016/j.ijhcs.2015.09.004]

36. Huberty J, Matthews J, Leiferman J, Cacciatore J, Gold K. A study protocol of a three-group randomized feasibility trial of an online yoga intervention for mothers after stillbirth (The Mindful Health Study). Pilot Feasibility Stud 2018;4(1):12. [doi: 10.1186/s40814-017-0162-7] [Medline: 28694991]

37. Cohen J. Statistical Power Analysis for the Behavioral Sciences. United Kingdom: Routledge; 1988.

38. Economides M, Martman J, Bell MJ, Sanderson B. Improvements in stress, affect, and irritability following brief use of a mindfulness-based smartphone app: a randomized controlled trial. Mindfulness (N Y) 2018;9(5):1584-1593 [REEE Full text] [doi: 10.1007/s12671-018-0905-4] [Medline: $\underline{\text { 30294390] }}$

39. Bostock S, Crosswell AD, Prather AA, Steptoe A. Mindfulness on-the-go: Effects of a mindfulness meditation app on work stress and well-being. J Occup Health Psychol 2019 Feb;24(1):127-138. [doi: 10.1037/ocp0000118] [Medline: 29723001]

40. Huberty J, Eckert R, Larkey L, Mesa R. Myeloproliferative neoplasm patients experience of using a consumer-based mobile meditation app to improve fatigue: informing future directions. J Med Internet Res 2019 (forthcoming)(forthcoming). 
41. Bakker D, Kazantzis N, Rickwood D, Rickard N. Mental health smartphone apps: review and evidence-based recommendations for future developments. JMIR Ment Health 2016;3(1):e7 [FREE Full text] [doi: 10.2196/mental.4984] [Medline: 26932350]

42. Newman M, Lauterbach D, Munson S, Resnick P, Morris M. It's not that i don't have problems, i'm just not putting them on facebook: Challenges and opportunities in using online social networks for health. In: Proceedings of the ACM 2011 conference on Computer supported cooperative work. 2011 Presented at: Conference on Computer Supported Cooperative Work; March 19-23, 2011; China p. 341-350. [doi: 10.1145/1958824.1958876]

43. Richardson CR, Buis LR, Janney AW, Goodrich DE, Sen A, Hess ML, et al. An online community improves adherence in an internet-mediated walking program. Part 1: results of a randomized controlled trial. J Med Internet Res 2010;12(4):e71 [FREE Full text] [doi: 10.2196/jmir.1338] [Medline: 21169160]

44. Ledesma D, Kumano H. Mindfulness-based stress reduction and cancer: a meta-analysis. Psychooncology 2009 Jun;18(6):571-579. [doi: 10.1002/pon.1400] [Medline: 19023879]

45. Carlson LE. Mindfulness-based interventions for coping with cancer. Ann N Y Acad Sci 2016 Jun;1373(1):5-12. [doi: 10.1111/nyas.13029] [Medline: 26963792]

46. Ross A, Thomas S. The health benefits of yoga and exercise: a review of comparison studies. J Altern Complement Med 2010 Jan;16(1):3-12. [doi: 10.1089/acm.2009.0044] [Medline: 20105062]

47. Schellekens MP, Tamagawa R, Labelle LE, Speca M, Stephen J, Drysdale E, et al. Mindfulness-Based Cancer Recovery (MBCR) versus Supportive Expressive Group Therapy (SET) for distressed breast cancer survivors: evaluating mindfulness and social support as mediators. J Behav Med 2017 Jun;40(3):414-422 [FREE Full text] [doi: 10.1007/s10865-016-9799-6] [Medline: 27722908]

48. Speca M, Carlson L, Mackenzie M. Mindfulness-based cancer recovery: An adaptation of Mindfulness-Based Stress Reduction (MBSR) for cancer patients. In: Baer R, editor. Mindfulness-Based Treatment Approaches. 2nd Edition. San Diego, CA: Elsevier; 2014:293-316.

49. Price GL, Davis KL, Karve S, Pohl G, Walgren RA. Survival patterns in United States (US) medicare enrollees with non-CML myeloproliferative neoplasms (MPN). PLoS One 2014;9(3):e90299 [FREE Full text] [doi: 10.1371/journal.pone.0090299] [Medline: 24618579]

50. Hui D, Zhukovsky D, Bruera E. Which treatment is better? Ascertaining patient preferences with crossover randomized controlled trials. J Pain Symptom Manage 2015;49(3):625-631. [Medline: 25555446]

51. Raghunathan NJ, Korenstein D, Li QS, Tonorezos ES, Mao JJ. Determinants of mobile technology use and smartphone application interest in cancer patients. Cancer Med 2018 Nov;7(11):5812-5819 [FREE Full text] [doi: 10.1002/cam4.1660] [Medline: 30280495]

52. Girault A, Ferrua M, Lalloué B, Sicotte C, Fourcade A, Yatim F, et al. Internet-based technologies to improve cancer care coordination: current use and attitudes among cancer patients. Eur J Cancer 2015 Mar;51(4):551-557. [doi: 10.1016/j.ejca.2014.12.001] [Medline: 25661828]

\title{
Abbreviations
}

MPN: myeloproliferative neoplasm

MPN-SAF: Myeloproliferative Neoplasm Symptom Assessment Form

NIH PROMIS: National Institutes of Health Patient Reported Outcomes Measurement Information System

RCT: randomized controlled trial

\author{
Edited by G Eysenbach; submitted 31.10.18; peer-reviewed by Y Zhang, L Morrison, $W$ van Harten, U Subnis, C Jacob; comments \\ to author 27.12.18; revised version received 11.02.19; accepted 24.03.19; published 29.04.19 \\ Please cite as: \\ Huberty J, Eckert R, Larkey L, Kurka J, Rodríguez De Jesús SA, Yoo W, Mesa R \\ Smartphone-Based Meditation for Myeloproliferative Neoplasm Patients: Feasibility Study to Inform Future Trials \\ JMIR Form Res 2019;3(2):e12662 \\ URL: http://formative.jmir.org/2019/2/e12662/ \\ doi: $\underline{10.2196 / 12662}$ \\ PMID: 31033443
}

CJennifer Huberty, Ryan Eckert, Linda Larkey, Jonathan Kurka, Sue A Rodríguez De Jesús, Wonsuk Yoo, Ruben Mesa. Originally published in JMIR Formative Research (http://formative.jmir.org), 29.04.2019. This is an open-access article distributed under the terms of the Creative Commons Attribution License (https://creativecommons.org/licenses/by/4.0/), which permits unrestricted use, distribution, and reproduction in any medium, provided the original work, first published in JMIR Formative Research, is 
properly cited. The complete bibliographic information, a link to the original publication on http://formative.jmir.org, as well as this copyright and license information must be included. 